\title{
Evaluation of Integrals of Howland Type Involving a Bessel Function
}

\author{
By Chih-Bing Ling and Ming-Jing Wu
}

\begin{abstract}
This paper presents a method of evaluation of four integrals of Howland type, which involve a Bessel function in the integrands. With the aid of tabulated values, they are evaluated to 10D. Two of the four Howland integrals needed in the evaluation are evaluated anew to $20 \mathrm{D}$ in order to provide adequate accuracy.
\end{abstract}

In a recent investigation of certain problems in elasticity concerning elliptic boundaries, four integrals of Howland type involving an additional Bessel function in the integrands were encountered. We believe that they deserve special consideration. The integrals are as follows:

$$
\begin{aligned}
& \begin{array}{l}
F_{n, k}(a)=\frac{2^{k}}{k !} \int_{0}^{\infty} \frac{m^{k} J_{n}(m a)}{\sinh 2 m \pm 2 m} d m \quad \begin{array}{l}
(n+k \geqslant 1), \\
F_{n, k}^{*}
\end{array}(n+k \geqslant 3),
\end{array}
\end{aligned}
$$

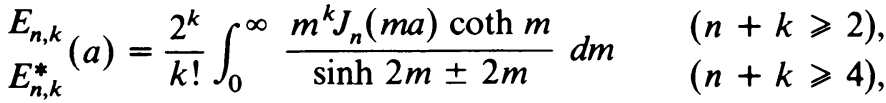

where $J_{n}$ is a Bessel function of the first kind of integral order $n . n$ and $k$ are nonnegative integers restricted as indicated above in order to render each integral convergent at the lower limit. The constant $a$ may be real or complex.

By using the usual series expression for $J_{n}$ and integrating, the first integral becomes

$$
F_{n, k}(a)=\sum_{p=0}^{\infty}(-1)^{p}\left(\begin{array}{c}
n+2 p \\
p
\end{array}\right)\left(\begin{array}{c}
n+k+2 p \\
k
\end{array}\right)\left(\frac{a}{4}\right)^{n+2 p} I_{n+k+2 p},
$$

where

$$
I_{k}=\frac{2^{k}}{k !} \int_{0}^{\infty} \frac{m^{k} d m}{\sinh 2 m+2 m} \quad(k \geqslant 1)
$$

Since $I_{k}$ tends asymptotically to unity as $k$ tends to infinity, we write, with a view of improving convergence of the series,

$$
\begin{aligned}
F_{n, k}(a)= & K_{n, k}(a) \\
& -\sum_{p=0}^{\infty}(-1)^{p}\left(\begin{array}{c}
n+2 p \\
p
\end{array}\right)\left(\begin{array}{c}
n+k+2 p \\
k
\end{array}\right)\left(1-I_{n+k+2 p}\right)\left(\frac{a}{4}\right)^{n+2 p},
\end{aligned}
$$

Received February 10, 1981; revised May 29, 1981.

1980 Mathematics Subject Classification. Primary 65A05, 65D20, 65D30.

Key words and phrases. Howland integrals, integrals involving Bessel function.

(c) 1982 American Mathematical Society 002 5-5 718/82/0000-0482/\$03.00 
where [7]

$$
\begin{aligned}
K_{n, k}(a) & =\sum_{p=0}^{\infty}(-1)^{p}\left(\begin{array}{c}
n+2 p \\
p
\end{array}\right)\left(\begin{array}{c}
n+k+2 p \\
k
\end{array}\right)\left(\frac{a}{4}\right)^{n+2 p} \\
& =\frac{2^{k+1}}{k !} \int_{0}^{\infty} m^{k} e^{-2 m} J_{n}(m a) d m=\frac{(n+k) !}{k !} P_{k}^{-n}(t) t^{k+1},
\end{aligned}
$$

in which $P_{k}^{-n}$ is an associated Legendre function of the first kind [1] and

$$
t=2 /\left(a^{2}+4\right)^{1 / 2} \text {. }
$$

Or, in terms of Gauss' hypergeometric function,

$$
K_{n, k}(a)=\left(\begin{array}{c}
n+k \\
k
\end{array}\right)\left(\frac{1-t}{1+t}\right)^{n / 2} t_{2}^{k+1} F_{1}\left(-k, k+1 ; n+1 ; \frac{1}{2}-\frac{t}{2}\right),
$$

if $t$ is real and $-1<t<1$. On the other hand, if $t$ is complex, the factor $(1-t)^{n / 2}$ is replaced by $(t-1)^{n / 2}$.

Similarly, the other three integrals are

$$
\begin{aligned}
F_{n, k}^{*}(a)= & K_{n, k}(a) \\
& +\sum_{p=0}^{\infty}(-1)^{p}\left(\begin{array}{c}
n+2 p \\
p
\end{array}\right)\left(\begin{array}{c}
n+k+2 p \\
k
\end{array}\right)\left(I_{n+k+2 p}^{*}-1\right)\left(\frac{a}{4}\right)^{n+2 p} \\
E_{n, k}(a)= & K_{n, k}(a) \\
& -\sum_{p=0}^{\infty}(-1)^{p}\left(\begin{array}{c}
n+2 p \\
p
\end{array}\right)\left(\begin{array}{c}
n+k+2 p \\
k
\end{array}\right)\left(1-I V_{n+k+2 p}\right)\left(\frac{a}{4}\right)^{n+2 p} \\
E_{n, k}^{*}(a)= & K_{n, k}(a) \\
& +\sum_{p=0}^{\infty}(-1)^{p}\left(\begin{array}{c}
n+2 p \\
p
\end{array}\right)\left(\begin{array}{c}
n+k+2 p \\
k
\end{array}\right)\left(I V_{n+k+2 p}^{*}-1\right)\left(\frac{a}{4}\right)^{n+2 p}
\end{aligned}
$$

where

$$
\begin{array}{cl}
I_{k}^{*} & =\frac{2^{k}}{k !} \int_{0}^{\infty} \frac{m^{k} d m}{\sinh 2 m-2 m} \quad(k \geqslant 3), \\
I V_{k} & =\frac{2^{k}}{k !} \int_{0}^{\infty} \frac{m^{k} \operatorname{coth} m d m}{\sinh 2 m \pm 2 m} \quad(k \geqslant 2), \\
I V_{k}^{*} & (k \geqslant 4) .
\end{array}
$$

The evaluation of the last two integrals in (9) has been considered before by the first author [4]. It was found that

$$
\begin{aligned}
& I V_{k}=I_{k}-\left(I_{k-1}+I I_{k-1}-S_{k}\right) / k, \\
& I V_{k}^{*}=I_{k}^{*}+\left(I_{k-1}^{*}+I I_{k-1}^{*}-S_{k}\right) / k,
\end{aligned}
$$

where

$$
\begin{aligned}
I I_{k} & =\frac{2^{k}}{k !} \int_{0}^{\infty} \frac{m^{k} e^{-2 m} d m}{\sinh 2 m \pm 2 m} \quad \begin{array}{l}
(k \geqslant 1), \\
I I_{k}^{*}
\end{array} \\
S_{k} & =\sum_{n=1}^{\infty} \frac{1}{n^{k}} \quad(k \geqslant 2) .
\end{aligned}
$$


The integrals $I_{k}, I_{k}^{*}, I I_{k}$ and $I I_{k}^{*}$ are the four ordinary Howland integrals. The first two were tabulated by the first author and Lin to 25D [6], [5] and the remaining two by Nelson to 9D [8]. The Riemann zeta function $S_{k}$ was tabulated by Glaisher for integral $k$ to 32D [2]. Among these tabulated values, it appears that Nelson's 9D values of $I I_{k}$ and $I I_{k}^{*}$ are inadequate for the present purpose. Consequently, the values are computed anew. The evaluation of these two integrals is described in the Appendix. Table 1 shows the values to 20D.

The relations in (4) and (8) are suitable for numercial computation. With the tabulated values, the four integrals can therefore be evaluated when $a$ is given. In case of slow convergence of the series, the Euler transformation for alternating series [3] may be applied. The following recurrence relation for the first integral is mentioned:

$$
F_{n-1, k}(a)+F_{n+1, k}(a)=\frac{4 n}{k a} F_{n, k-1}(a),
$$

which is derived by virtue of the relation connecting three Bessel functions of consecutive integral orders. Similar relations can be found for the other three integrals as well as for $K_{n, k}(a)$. These recurrence relations may be used for checking purposes. They can also be used for computing an unknown integral from two known integrals.

Values of the four integrals for the particular value $a=1$ are computed. The expression in (7) is used for computing $K_{n, k}$. The results for $n=0(1) 3$ and $k=0(1) 10$ are shown in Table 2 to 10D.

Appendix. Evaluation of $I I_{k}$ and $I I_{k}^{*}$. The two Howland integrals in (11) may be written as

$$
\begin{aligned}
& I I_{k}=\frac{1}{2(k !)} \int_{0}^{\infty} \frac{w^{k} e^{-w} d w}{\sinh w \pm w} \quad(k \geqslant 1), \\
& (k \geqslant 3) .
\end{aligned}
$$

Expansion of the integrand yields

$$
\frac{w^{k} e^{-w}}{\sinh w \pm w}=\frac{2 w^{k} e^{-2 w}}{1 \pm 2 w e^{-w}-e^{-2 w}}=2 w^{k} e^{-2 w} \sum_{n=0}^{\infty}(\mp 1)^{n} p_{n}(w) e^{-n w}
$$

where $p_{n}(w)$ is a polynomial in $w$ of degree $n$ related to the Gegenbauer polynomial of order unity. Its form is different according as $n$ is even or odd. For $n \geqslant 0$,

$$
p_{2 n}(w)=\sum_{t=0}^{n}\left(\begin{array}{c}
n+t \\
2 t
\end{array}\right)(2 w)^{2 t}, \quad p_{2 n+1}(w)=\sum_{t=0}^{n}\left(\begin{array}{c}
n+t+1 \\
2 t+1
\end{array}\right)(2 w)^{2 t+1}
$$

With the aid of the integral

$$
\int_{0}^{\infty} w^{t} e^{-c w} d w=\frac{t !}{c^{t+1}} \quad(c>0)
$$

we get

$$
I I_{k}=\sum_{n=1}^{\infty}(\mp 1)^{n+1} \frac{q_{n}(k)}{(n+1)^{k+1}}
$$


where, for $n \geqslant 0$,

$$
\begin{aligned}
& q_{2 n+1}(k)=\sum_{t=0}^{n}\left(\begin{array}{c}
k+2 t \\
k
\end{array}\right) \frac{(n+t) !}{(n-t) !} \frac{1}{(n+1)^{2 t}} \\
& q_{2 n+2}(k)=\sum_{t=0}^{n}\left(\begin{array}{c}
k+2 t+1 \\
k
\end{array}\right) \frac{(n+t+1) !}{(n-t) !}\left(\frac{2}{2 n+3}\right)^{2 t+1} .
\end{aligned}
$$

The series in (17) is rapidly convergent when $k$ is large but slowly convergent when $k$ is small. For instance, an accuracy of 20D can be reached with the first six terms when $k \geqslant 30$, with the first twenty terms when $k \geqslant 22$, and with the first eighty terms when $k \geqslant 18$. To reach an accuracy of $25 \mathrm{D}$, the corresponding values of $k$ are not less than 35,26 , and 21 , respectively.

It is noted that these two integrals, unlike $I_{k}$ and $I_{k}^{*}$, cannot be evaluated by Plana's method. In terms of $I_{k}$ and $I_{k}^{*}$, the following relations are obtained by expanding $e^{-w}$ into series of $w$ and then applying the Kummer transformation [3]:

$$
\begin{aligned}
& \frac{1}{2^{k+1}}-I I_{k}=\sum_{n=0}^{\infty}(-1)^{n}\left(\begin{array}{c}
n+k \\
n
\end{array}\right)\left(1-I_{n+k}\right), \\
& I I_{k}^{*}-\frac{1}{2^{k+1}}=\sum_{n=0}^{\infty}(-1)^{n}\left(\begin{array}{c}
n+k \\
n
\end{array}\right)\left(I_{n+k}^{*}-1\right) .
\end{aligned}
$$

They are suitable for computation only for the first few values of $k$. When $k$ increases, accuracy is rapidly lost due to the binomial coefficient involved in the series. However, it appears that the loss can be reduced if the computation is carried out in several steps through some intermediate integrals. Suppose that $s$ steps are taken and in each step a factor $e^{-w / s}$ is expanded into series instead of $e^{-w}$. Denote the intermediate integrals in the $r$ th step by

$$
\begin{array}{ll}
I_{k}^{(r)} & =\frac{1}{2(k !)} \int_{0}^{*(r)} \frac{w^{k} e^{-r w s} d w}{\sinh w \pm w} \quad(k \geqslant 1), \\
& (k \geqslant 3),
\end{array}
$$

where $r=0,1,2, \ldots, s$. These expressions give $I_{k}$ and $I_{k}^{*}$ when $r=0$, and $I I_{k}$ and $I I_{k}^{*}$ when $r=s$. In a similar manner, it is found that

$$
\begin{aligned}
& \left(\frac{s}{s+r}\right)^{k+1}-I_{k}^{(r)}=\sum_{n=0}^{\infty} \frac{(-1)^{n}}{s^{n}}\left(\begin{array}{c}
n+k \\
n
\end{array}\right)\left\{\left(\frac{s}{s+r-1}\right)^{n+k+1}-I_{n+k}^{(r-1)}\right\} \\
& I_{k}^{*(r)}-\left(\frac{s}{s+r}\right)^{k+1}=\sum_{n=0}^{\infty} \frac{(-1)^{n}}{s^{n}}\left(\begin{array}{c}
n+k \\
n
\end{array}\right)\left\{I_{n+k}^{*(r-1)}-\left(\frac{s}{s+r-1}\right)^{n+k+1}\right\} .
\end{aligned}
$$

It is seen that the binomial coefficient involved in the series is now divided by a factor $s^{n}$. Hence, the loss of accuracy is considerably reduced, especially when $s$ is large. If the computation is carried out recurrently $s$ times, the integrals $I I_{k}$ and $I I_{k}^{*}$ are obtained. Also, it turns out that the total loss of accuracy in $s$ steps together is smaller than that by (19) in a single step. For instance, when $k=20$, the total loss is $10 \mathrm{~S}$ if $s=2,7 \mathrm{~S}$ if $s=4$, and $5 \mathrm{~S}$ if $s=7$. The loss is less when $k$ is smaller. On the other hand, the computing time required for $s$ steps is increased $s$ times. Furthermore, analogous to (17), the expansions of the intermediate integrals in the $r$ th step are

$$
I_{k}^{*(r)}=\sum_{n=1}^{\infty}(\mp 1)^{n+1} q_{n}^{(r)}(k)\left(\frac{s}{n s+r}\right)^{k+1}
$$


where, for $n \geqslant 0$,

$$
\begin{aligned}
& q_{2 n+1}^{r)}(k)=\sum_{t=0}^{n}\left(\begin{array}{c}
k+2 t \\
k
\end{array}\right) \frac{(n+t) !}{(n-t) !}\left\{\frac{2 s}{(2 n+1) s+r}\right\}^{2 t}, \\
& q_{2 n+2}^{(r)}(k)=\sum_{t=0}^{n}\left(\begin{array}{c}
k+2 t+1 \\
k
\end{array}\right) \frac{(n+t+1) !}{(n-t)}\left\{\frac{2 s}{(2 n+2) s+r}\right\}^{2 t+1} .
\end{aligned}
$$

Note that when $r=s$, these expressions become those for $I I_{k}$ and $I I_{k}^{*}$ as given in (17) and (18). Suppose that in the present computation, an accuracy not less than $20 \mathrm{D}$ is prescribed to the values of $I I_{k}$ and $I I_{k}^{*}$. With the 25D values of $I_{k}$ and $I_{k}^{*}$, the loss of accuracy must therefore be kept within 5S. In each step, the relations in (21) with $s=7$ are used for $k$ up to 20. For larger $k$, the relations in (22) are used instead. The values are also computed by both methods for some overlapping values of $k$ to serve as a check. Further checks on the final results are provided by the following relations:

$$
\begin{aligned}
& \sum_{k=0}^{\infty} I I_{2 k+1}=\frac{1}{2}-I I_{1}, \quad \sum_{k=1}^{\infty} k I_{2 k}=\frac{1}{4}-I I_{2}, \\
& \sum_{k=1}^{\infty} I I_{2 k+1}^{*}=\frac{1}{2}, \quad \sum_{k=2}^{\infty} k I I_{2 k}^{*}=\frac{1}{4} \text {. }
\end{aligned}
$$

The values rounded to $20 \mathrm{D}$ are shown in Table 1 . No discrepancy is noticed in Nelson's 9D values when they are compared with the present values.

Corrigenda. The factor $(-1)^{m+n}$ in (3) and (7) of the paper [6] and also in (3) of the paper [5] should be deleted. The expressions (6) in the paper [6] should be revised accordingly. These corrections, however, do not effect the numerical results

\begin{tabular}{|c|c|c|c|c|c|c|c|c|}
\hline $\mathrm{k}$ & \multicolumn{4}{|c|}{$\mathrm{II}_{\mathrm{k}}$} & \multicolumn{4}{|c|}{$\mathrm{II}_{\mathrm{k}}^{*}$} \\
\hline 1 & 0.22011 & 95814 & 42489 & 13267 & & - & & \\
\hline 2 & 0.08792 & 72351 & 54623 & 64461 & & - & & \\
\hline 3 & 0.04334 & 78620 & 32253 & 03164 & 0.46071 & 37190 & 35659 & 66356 \\
\hline 4 & 0.02258 & 30042 & 91563 & 34696 & 0.09931 & 55321 & 31928 & 69508 \\
\hline 5 & 0.01192 & 34729 & 94286 & 16469 & 0.03241 & 26902 & 77966 & 60244 \\
\hline 6 & 0.00628 & 79721 & 18696 & 33642 & 0.01261 & 69084 & 78437 & 55392 \\
\hline 7 & 0.00329 & 50133 & 54822 & 42863 & 0.00539 & 11167 & 37422 & 13901 \\
\hline 8 & 0.00171 & 32982 & 31483 & 05028 & 0.00243 & 29999 & 83832 & 20264 \\
\hline 9 & 0.00088 & 41479 & 97290 & 09130 & 0.00113 & 59968 & 30348 & 45397 \\
\hline 10 & 0.00045 & 32145 & 74349 & 76403 & 0.00054 & 22017 & 96217 & 86263 \\
\hline 11 & 0.00023 & 10097 & 74078 & 29735 & 0.00026 & 25896 & 85716 & 8921 \\
\hline 12 & 0.00011 & 72094 & 40904 & 63806 & 0.00012 & 84309 & 57923 & 71136 \\
\hline 13 & 0.00005 & 92535 & 02802 & 31223 & 0.00006 & 32389 & 09903 & 71072 \\
\hline 14 & 0.00002 & 98701 & 48236 & 51008 & 0.00003 & 12834 & 43520 & 328 \\
\hline 15 & 0.00001 & 50252 & 72536 & 49084 & 0.00001 & 55254 & 16440 & 55194 \\
\hline 16 & 0.00000 & 75457 & 13757 & 46890 & 0.00000 & 77222 & 95797 & 22959 \\
\hline 17 & 0.00000 & 37848 & 78742 & 55501 & 0.00000 & 38470 & 73132 & 06282 \\
\hline 18 & 0.00000 & 18967 & 75182 & 64532 & 0.00000 & 19186 & 37 & 52976 \\
\hline 19 & 0.00000 & 09499 & 41039 & 13811 & 0.00000 & 09576 & 01987 & 42877 \\
\hline 20 & 0.00000 & 04755 & 23929 & 87590 & 0.00000 & 04782 & 03676 & 61263 \\
\hline
\end{tabular}
of the Howland integrals $I_{k}$ and $I_{k}^{*}$ in these two papers.

\section{TABLE I}

Values of $I I_{k}$ and $I I_{k}^{*}$ 
TABLE I (continued)

\begin{tabular}{|c|c|c|c|c|c|c|c|c|}
\hline $\mathrm{k}$ & & $\mathrm{II}_{\mathrm{k}}$ & & & & $\mathrm{II}_{\mathrm{k}}^{*}$ & & \\
\hline 21 & 0.00000 & 02379 & 58146 & 32115 & 0.00000 & 02388 & 93568 & 1874 \\
\hline 22 & 0.00000 & 01190 & 48321 & 96864 & 0.00000 & 01193 & 74219 & 70259 \\
\hline 23 & 0.00000 & 00595 & 48513 & 89530 & 0.00000 & 00596 & 61850 & 89582 \\
\hline 24 & 0.00000 & 00297 & 82793 & 38128 & 0.00000 & 00298 & 22142 & 27687 \\
\hline 25 & 0.00000 & 00148 & 94380 & 42812 & 0.00000 & 00149 & 08020 & 42756 \\
\hline 26 & 0.00000 & 00074 & 48230 & 48966 & 0.00000 & 00074 & 52951 & 81493 \\
\hline 27 & 0.00000 & 00037 & 24477 & 12334 & 0.00000 & 00037 & 26109 & 13887 \\
\hline 28 & 0.00000 & 00018 & 62364 & 19592 & 0.00000 & 00018 & 62927 & 61929 \\
\hline 29 & 0.00000 & 00009 & 31225 & 63641 & 0.00000 & 00009 & 31419 & 91799 \\
\hline 30 & 0.00000 & 00004 & 65627 & 88197 & 0.00000 & 00004 & 65694 & 80066 \\
\hline 31 & 0.00000 & 00002 & 32819 & 145 & 000 & 00002 & 32842 & 1708 \\
\hline 32 & 0.00000 & 00001 & 16411 & 36812 & 0.00000 & 00001 & 16419 & 28317 \\
\hline 33 & 0.00000 & 00000 & 58206 & 30278 & 0.00000 & 00000 & 58209 & 02107 \\
\hline 34 & 0.00000 & 00000 & 29103 & 36435 & 0.00000 & 00000 & 29104 & 29710 \\
\hline 35 & 0.00000 & 00000 & 14551 & 75540 & 0.00000 & 00000 & 14552 & 07520 \\
\hline 36 & 0.00000 & 00000 & 07275 & 90285 & 0.00000 & 00000 & 07276 & 0124 \\
\hline 37 & 0.00000 & 00000 & 03637 & 96006 & 0.00000 & 00000 & 03637 & $9975^{\circ}$ \\
\hline 38 & 000 & 00000 & 01818 & 98299 & 0000 & 00000 & 01818 & 9958 \\
\hline 39 & 0.00000 & 00000 & 00909 & 49 & 0000 & 00000 & 00909 & 4969 \\
\hline 40 & 0.00000 & 00000 & 00454 & 746 & 0.00000 & 00000 & 00454 & 7481 \\
\hline 41 & 00 & 00000 & 00227 & & 000 & 00000 & 00227 & 373 \\
\hline 42 & 0.0 & 00000 & 00113 & 686 & 0000 & 00000 & 00113 & 6869 \\
\hline 43 & 0.00000 & 00000 & 00056 & 843 & 000 & 00000 & 00056 & 84345 \\
\hline 44 & 0.00000 & 00000 & 00028 & 42170 & 000 & 00000 & 00028 & 42172 \\
\hline 45 & 000 & 00000 & 00014 & 210 & 000 & 00000 & 00014 & 21086 \\
\hline 46 & 00 & 00000 & 00007 & 10 & 00 & 00000 & 00007 & 10543 \\
\hline 47 & 0 & 00000 & 000 & 55 & 0 & 00000 & 00003 & 55271 \\
\hline 48 & & 00 & 00 & 7 & & 00 & 01 & 536 \\
\hline 49 & & 00 & 00 & & & 00 & 00 & 8 \\
\hline 50 & 0.00000 & & 00 & & & & & \\
\hline 51 & & 00 & & 22 & & & & \\
\hline 52 & & & 00 & 11 & 00 & 00000 & 00 & 02 \\
\hline 53 & 00 & 00 & $00 c$ & 05 & 00 & 00000 & 00000 & $0555]$ \\
\hline 54 & 0.00000 & 00000 & 00000 & 02776 & 0.00000 & 00000 & 00000 & 0277 \\
\hline 55 & 0.00000 & 00000 & 00000 & 01388 & 0.00000 & 00000 & 00000 & 01388 \\
\hline 56 & 0.00000 & 00000 & 00000 & 00694 & 0.00000 & 00000 & 00000 & 00694 \\
\hline 57 & 0.00000 & 00000 & 00000 & 00347 & 0.00000 & 00000 & 00000 & 00347 \\
\hline 58 & 0.00000 & 00000 & 00000 & 0017 & 0.00000 & 00000 & 00000 & 00173 \\
\hline 59 & 0.00000 & 00000 & 00000 & 00087 & 0.00000 & 00000 & 00000 & 00087 \\
\hline 60 & 0.00000 & 00000 & 00000 & 00043 & 0.00000 & 00000 & 00000 & 00043 \\
\hline 61 & 0 & 00000 & 00000 & 0 & & 00000 & 00000 & 00022 \\
\hline 62 & 0.00000 & 00000 & 00000 & 00011 & 0.00000 & 00000 & 00000 & 00011 \\
\hline 63 & 0.00000 & 00000 & 00000 & 00005 & 0.00000 & 00000 & 00000 & 00005 \\
\hline 64 & 0.00000 & 00000 & 00000 & 00003 & 0.00000 & 00000 & 00000 & 00003 \\
\hline 65 & 0.00000 & 00000 & 00000 & 00001 & 0.00000 & 00000 & 00000 & 00001 \\
\hline 66 & 0.00000 & 00000 & 00000 & 00001 & 0.00000 & 00000 & 00000 & 00001 \\
\hline
\end{tabular}


TABLE 2

Values of four integrals for $a=1$

\begin{tabular}{|c|c|c|c|c|c|}
\hline $\mathbf{n}$ & $\mathrm{k}$ & $\mathrm{F}_{\mathrm{n}, \mathrm{k}}(1)$ & $\mathrm{F}_{\mathrm{n}, \mathrm{k}}^{*}(1)$ & $\mathrm{E}_{\mathrm{n}, \mathrm{k}}(1)$ & $E_{n, k}^{*}(1)$ \\
\hline \multirow[t]{10}{*}{0} & 1 & $0.54084 \quad 46591$ & - & - & - \\
\hline & 2 & 0.3419408513 & - & 0.6346934541 & - \\
\hline & 3 & 0.1879553763 & 1.1570758719 & 0.2756206093 & - \\
\hline & 4 & 0.0480162464 & 0.3381339129 & 0.0807815488 & 0.6459926848 \\
\hline & 5 & -0.0631663029 & 0.0410933656 & -0.0505494481 & 0.0996922234 \\
\hline & 6 & -0.1333910175 & -0.0967005792 & -0.1287407789 & -0.0813634422 \\
\hline & 7 & -0.1600474673 & -0.1490754405 & -0.1585154846 & -0.1447657105 \\
\hline & 8 & -0.1493352743 & -0.1475867013 & -0.1489497848 & -0.1464535865 \\
\hline & 9 & -0.1129926003 & -0.1139891334 & -0.1129769522 & -0.1137737369 \\
\hline & 10 & -0.0646118449 & -0.0660073063 & -0.0646826817 & -0.0660270468 \\
\hline \multirow[t]{11}{*}{1} & 0 & 0.1606918196 & - & - & - \\
\hline & 1 & $0.26110 \quad 17772$ & - & $0.41598 \quad 35324$ & - \\
\hline & 2 & $0.33007 \quad 03722$ & 1.1421161369 & 0.4056504816 & - \\
\hline & 3 & $0.34825 \quad 03408$ & 0.7205905605 & $0.39028 \quad 30431$ & 1.0505169033 \\
\hline & 4 & 0.3143077838 & $0.51523 \quad 39857$ & 0.3377173885 & 0.6027022003 \\
\hline & 5 & 0.2408951857 & 0.3512315466 & $0.25357 \quad 33417$ & 0.3832309369 \\
\hline & 6 & 0.1476405342 & $0.20700 \quad 47013$ & 0.1542600266 & 0.2200755146 \\
\hline & 7 & 0.0545561086 & $0.08540 \quad 39244$ & 0.0578780057 & 0.0909634111 \\
\hline & 8 & -0.0225470977 & -0.0071885853 & -0.0209479499 & -0.0048047337 \\
\hline & 9 & $-0.07432 \quad 31433$ & -0.0670520664 & -0.0735868206 & -0.0660411886 \\
\hline & 10 & -0.0981679533 & -0.0949309795 & -0.0978454429 & -0.0945133296 \\
\hline \multirow[t]{11}{*}{2} & 0 & $0.03700 \quad 02514$ & - & 0.0567284626 & - \\
\hline & 1 & 0.1019226191 & 0.3124728392 & $0.12168 \quad 33587$ & - \\
\hline & 2 & 0.1802627032 & 0.3310676512 & 0.1972736106 & $0.45768 \quad 54746$ \\
\hline & 3 & 0.2521384533 & 0.3657456439 & 0.2652466995 & 0.4120263376 \\
\hline & 4 & 0.3002340944 & 0.3824566476 & $0.30950 \quad 14944$ & $0.40452 \quad 42185$ \\
\hline & 5 & 0.3146125299 & $0.37109 \quad 38229$ & 0.3207233589 & 0.3824695368 \\
\hline & 6 & 0.2939878080 & 0.3308549436 & 0.2977896734 & $0.33685 \quad 07335$ \\
\hline & 7 & 0.2444134868 & 0.2673638413 & 0.2466640712 & $0.27052 \quad 31475$ \\
\hline & 8 & 0.1766133286 & 0.1902886636 & $0.17788 \quad 87876$ & $0.19193 \quad 52921$ \\
\hline & 9 & 0.1029716679 & 0.1107942066 & $0.10366 \quad 67523$ & 0.1116382998 \\
\hline & 10 & 0.0348825876 & $0.03918 \quad 64797$ & 0.0352479535 & $0.03961 \quad 05714$ \\
\hline \multirow[t]{11}{*}{3} & 0 & 0.0094063033 & 0.0272777106 & 0.0110903091 & - \\
\hline & 1 & $0.03490 \quad 02343$ & 0.0610133066 & $0.03784 \quad 41688$ & 0.0823646842 \\
\hline & 2 & $0.07762 \quad 01043$ & 0.1077752201 & $0.08108 \quad 29533$ & 0.1196776422 \\
\hline & 3 & $0.13245 \quad 02011$ & 0.1622565094 & 0.1357799185 & 0.1699776958 \\
\hline & 4 & 0.1899691228 & $0.21625 \quad 73021$ & 0.1927760106 & $0.22135 \quad 04748$ \\
\hline & 5 & 0.2394793654 & 0.2606990895 & 0.2416290493 & 0.2640078127 \\
\hline & 6 & 0.2718428389 & $0.28778 \quad 70626$ & 0.2733711186 & $0.28988 \quad 38678$ \\
\hline & 7 & 0.2814299577 & 0.2927160111 & 0.2824530496 & $0.29400 \quad 88557$ \\
\hline & 8 & 0.2669605846 & $0.27455 \quad 24265$ & 0.2676120212 & 0.2753278811 \\
\hline & 9 & $0.23131 \quad 27687$ & $0.23619 \quad 75451$ & $0.23171 \quad 01874$ & $0.23665 \quad 03371$ \\
\hline & 10 & 0.1805452877 & $0.18356 \quad 63448$ & 0.1807788447 & 0.1838239694 \\
\hline
\end{tabular}

Dpartment of Mathematics

Virginia Polytechnic Institute and State University

Blacksburg, Virginia 24061

Institute of Mathematics

Academia Sinica

Taipei, Taiwan, Republic of China 
1. A. ERdély et AL., Higher Transcendental Functions, vol. 1, Chapter 3, McGraw-Hill, New York, 1953.

2. J. W. L. Glaisher, "Tables of $1 \pm 2^{-n}+3^{-n} \pm 4^{-n}+$ etc., etc. to 32 places of decimals," Quart. J. Math., v. 45, 1914, pp. 141-158.

3. K. KNOPP, Infinite Series, Hafner, New York, 1947.

4. C. B. LnG, "Tables of values of 16 integrals of algebraic-hyperbolic type," MTAC., v. 11, 1957, pp. $160-166$.

5. C. B. Ling, "Further evaluation of Howland integrals," Math. Comp., v. 32, 1978, pp. 900-904.

6. C. B. LiNG \& J. LIN, "A new method of evaluation of Howland integrals," Math. Comp., v. 25, 1971, pp. 331-337.

7. Y. L. LUKe, Integrals of Bessel Functions, McGraw-Hill, New York, 1962.

8. C. W. Nelson, "New tables of Howland's and related integrals," Math. Comp., v. 15, 1961, pp. 12-18. 